\title{
Modelos para dimensionamento de pavimentos de concreto simples submetidos a carregamentos rodoviários e ambientais empregando análise multivariada de dados
}

\author{
Marcos Paulo Rodolfo ${ }^{1}$; José Tadeu Balbo²
}

\begin{abstract}
Resumo: Diversos métodos modernos de dimensionamento de pavimentos em concreto de cimento Portland são desenvolvidos a partir da modelagem estatística das tensões que solicitam essas estruturas. Utilizando um projeto fatorial que abrange dimensões estruturais largamente adotadas em projetos brasileiros, os autores lançaram mão do método dos elementos finitos (MEF) para calcular as tensões decorrentes do efeito conjunto de um eixo padrão de $80 \mathrm{kN}$ e de gradientes térmicos ambientais obtidos em pistas de teste brasileiras. Essas tensões foram trabalhadas sob a ótica da análise multivariada de dados, que é explicitada neste artigo. São propostos dois modelos de cálculo das tensões principais em placas de concreto, apoiadas em bases cimentadas ou bases granulares, que apresentaram resultados estatisticamente idênticos ao MEF original. Por fim é realizada uma aplicação dos modelos nacionais à estrutura de pavimento utilizada na nova pista da Rodovia dos Imigrantes em 2002 que havia sido dimensionada por critério não oficial estrangeiro.
\end{abstract}

\begin{abstract}
Several modern concrete pavement design guides are developed from the statistical modeling of the stresses found in those structures. Using a concrete pavement structures factorial project that considers widely adopted dimensions for slabs in Brazil, the authors applied the finite element method (FEM) to compute stresses caused by the joint effect of single axle loads of $80 \mathrm{kN}$ and environmental thermal gradients obtained from Brazilian road tests. Those stresses were analyzed by the multivariate data analysis concepts formally presented herein. The authors present two models to compute principal stresses in concrete slabs, supported by unbounded cemented bases or by granular bases. The use of such equations results in statistically identical stresses to those computed by original FEM. At last, an application of such new models to the concrete pavement design of the former 2002 construction of Imigrantes Highway are presented and compared to the original design using a non official alien method.
\end{abstract}

\section{INTRODUÇÃO}

Os efeitos climáticos, como empenamentos térmicos ou hidráulicos, não são tradicionalmente levados em consideração de maneira explícita no dimensionamento de pavimentos de concreto. Hall (2000) recomendava que, no futuro, tais questões fossem consideradas. Rodolfo e Balbo (2009) publicaram um conjunto de equações dedicadas ao cálculo de tensões em pavimentos (placas) de concreto rodoviárias. Este trabalho apresenta os fundamentos teórico-estatísticos empregados na formulação desses novos modelos bem como cotejá-los à luz de um exemplo real de dimensionamento e construção amplamente divulgado na literatura pertinente ao assunto no Brasil.

Os modelos estatísticos são ferramentas presentes há muito tempo no quotidiano dos projetistas de rodovias e que, com o crescente aumento no desempenho dos recursos computacionais, tiveram sua produção incentivada. $\mathrm{O}$ objetivo de um modelo estatístico não é apresentar uma solução matemática idêntica àquela desenvolvida pela análise clássica das estruturas, mas sim propor uma solução simples que possa ser utiliza-

\footnotetext{
${ }^{1}$ Marcos Paulo Rodolfo, Escola Politécnica, Universidade de São Paulo, São Paulo, SP, Brasil. (e-mail: eng.mprodolfo@gmail.com).

${ }^{2}$ José Tadeu Balbo, Escola Politécnica, Universidade de São PauIo, São Paulo, SP, Brasil. (e-mail: jotbalbo@usp.br).
}

Manuscrito recebido em 12/6/2010 e aprovado para publicação em 7/7/2010. Este artigo é parte de TRANSPORTES, volume XVIII, número 3, setembro de 2010. ISSN: 2237-1346 (online). da com confiabilidade dentro de intervalos predefinidos de variação de seus parâmetros de entrada. Esta é uma maneira comum de se configurar equações de projeto simplificadas. Dessa forma, por sua facilidade de uso e precisão, podem substituir modelos mais complexos nas fases preliminares de um projeto, permitindo a simulação de vários cenários, ou mesmo tornarem-se parte de métodos de dimensionamento (NCHRP, 2003; PCA, 1984) ou normatizações (PMSP, 2004).

Os modelos apresentados nesse trabalho poderão ser, no futuro, incorporados num novo método nacional de dimensionamento de pavimentos de concreto simples, levando em consideração as diferentes configurações de cargas (ambientais e rodoviárias) encontradas nas estradas e rodovias brasileiras. Os conceitos estatísticos discutidos têm condições de serem adotados em outros estudos, ainda mais completos, de pavimentos industriais, aeroportuários e portuários. Adotando os melhores métodos de dimensionamento de pavimentos, quer sejam flexíveis ou rígidos, os engenheiros brasileiros poderão no futuro adotar modelos decisórios sobre a melhor escolha do tipo de pavimento baseados na análise do custo do ciclo de vida da rodovia.

\subsection{Modelagem numérica por elementos finitos}

Avaliaram-se, nesse estudo, estruturas de pavimentos em concreto de cimento Portland submetidas a carre- 
gamentos rodoviários (cargas de rodas) e ambientais (gradientes térmicos). Os efeitos da ação do carregamento ambiental em pavimentos são de conhecimento dos engenheiros há muito tempo e foram descritos inicialmente por Westergaard (1927), como decorrentes da variação no volume da placa de concreto. Huang (2003) esclarece que essas variações são contidas pelo peso próprio da placa e pelo atrito entre a placa e a base. Tais condições ocasionam o surgimento de tensões de tração no fundo e de compressão no topo da placa, no caso em que a temperatura no topo é superior à temperatura de fundo, o chamado gradiente térmico positivo.

Diversos outros pesquisadores mediram os gradientes térmicos encontrados nas placas e desenvolveram modelos empíricos-estatísticos dessas medições. Balbo e Severi (2002) compararam seus estudos em ambiente tropical com outros realizados ao redor do mundo. Concluíram, que o gradiente térmico encontrado na cidade de São Paulo foi 30\% superior ao sugerido por Huang (1993) e 75\% superiores aos encontrados por Armaghani et al. (1987), ambos estudos em clima temperado. Em função da grande correlação entre o meio-ambiente e os gradientes térmicos observados, utiliza-se, neste trabalho, a faixa de gradientes observada por Balbo e Severi (2002), que varia entre $0^{\circ} \mathrm{C}$ e $25^{\circ} \mathrm{C}$.

Foram modelados pavimentos de concreto simples com barras de transferência de carga nas juntas transversais e barras de ligação nas juntas longitudinais apoiados em bases de concreto compactado com rolo (CCR) ou pavimentos apoiados diretamente em bases granulares. O carregamento rodoviário se deu por meio de um eixo simples de rodas duplas (ESRD) de $80 \mathrm{kN}$, posicionado no centro da borda longitudinal como apresentado por Huang e Wang (1973), adotado pelo método de projeto oficial dos EUA (NCHRP, 2003) e pela norma de projeto da PMSP (PMSP, 2004). Buscou-se abranger, no projeto fatorial, um longo espectro de estruturas, garantindo variações significativas dos parâmetros estruturais, como apre- sentados nas Tabelas 1 e 2 .

A modelagem por elementos finitos foi executada no programa ILSL2 (Khazanovich e Ioannides, 1993). A malha adotada nessa etapa, com 275 elementos, foi comparada com outra malha, com 1.556 elementos. $\mathrm{O}$ erro encontrado entre esses dois casos foi de apenas $1,1 \%$, assegurando que os erros inerentes à malha estavam sob controle. O resultado das simulações foi, então, trabalhado sob a ótica da análise multivariada de dados.

\section{ANÁLISE MULTIVARIADA DAS TENSÕES}

A análise multivariada de dados é baseada no princípio que envolve a observação e análise de mais de uma variável estatística. Cada dado é composto por um conjunto de variáveis e um objeto. O método é utilizado para efetuar estudos em múltiplas dimensões enquanto leva-se em consideração o efeito de todas as variáveis no objeto (Mardia et al., 1979). Diversas técnicas de análise estatística univariada podem ser aplicadas na análise multivariada: no caso discutido neste trabalho, técnicas de regressões simples são generalizadas para regressões múltiplas.

\subsection{Pavimentos com bases cimentadas}

Com posse do resultado das 4.950 simulações das estruturas com base cimentada oriundas do programa ILSL2 iniciou-se a análise estatística da tensão principal máxima observada na fibra inferior da placa $(\sigma)$ e sua relação entre os parâmetros envolvidos $\left(\mathrm{H}, \mathrm{H}_{\mathrm{BC}}\right.$, $\mathrm{E}_{\mathrm{CCR}}, \mathrm{k}$ e T).

\subsubsection{Análise da relação entre as variáveis}

Hair Jr. et al. (2005) apresentam que a utilização de gráficos de dispersão entre as diversas variáveis em estudo é uma maneira de se determinar se a relação entre elas é linear ou curvilínea (não-linear). O uso de gráficos tridimensionais, conforme demonstrado por Montgomery e Runger (2003) é outra maneira que pode auxiliar o pesquisador a avaliar, também, interação entre os diversos regressores, além da própria re-

Tabela 1. Parâmetros para os pavimentos com bases não-aderidas

\begin{tabular}{ll}
\hline Variável & Valores adotados \\
\hline Espessura da placa $(\mathrm{H})$ & de $0,15 \mathrm{~m}$ a $0,35 \mathrm{~m}$ \\
Módulo de elasticidade do concreto $\left(\mathrm{E}_{\mathrm{CCP}}\right)$ & $30 \mathrm{GPa}$ \\
Espessura da base cimentada $\left(\mathrm{H}_{\mathrm{BC}}\right)$ & de $0,10 \mathrm{~m}$ a $0,30 \mathrm{~m}$ \\
Módulo de elasticidade da base cimentada $\left(\mathrm{E}_{\mathrm{BC}}\right)$ & de $10 \mathrm{GPa} 30 \mathrm{GPa}$ \\
Módulo de reação do subleito $(\mathrm{k})$ & de $30 \mathrm{MPa} / \mathrm{m} \mathrm{a} 130 \mathrm{MPa} / \mathrm{m}$ \\
Diferencial térmico entre topo e fundo da placa (linear) $(\mathrm{T})$ & de $0{ }^{\circ} \mathrm{C} \mathrm{a} 25^{\circ} \mathrm{C}$ \\
\hline
\end{tabular}

Tabela 2. Parâmetros para os pavimentos com bases granulares

\begin{tabular}{ll}
\hline Variável & Valores adotados \\
\hline Espessura da placa $(\mathrm{H})$ & de $0,15 \mathrm{~m}$ a $0,35 \mathrm{~m}$ \\
Módulo de elasticidade do concreto $\left(\mathrm{E}_{\mathrm{CCP}}\right)$ & $30 \mathrm{GPa}$ \\
Módulo de reação do sistema de apoio $(\mathrm{k})$ & de $20 \mathrm{MPa} / \mathrm{m} \mathrm{a} 140 \mathrm{MPa} / \mathrm{m}$ \\
Diferencial térmico entre topo e fundo da placa (linear) $(\mathrm{T})$ & de $0{ }^{\circ} \mathrm{C} \mathrm{a} 25^{\circ} \mathrm{C}$ \\
\hline
\end{tabular}


lação entre os regressores e a variável dependente. Seguindo ambas as abordagens, avaliou-se as relações entre $\mathrm{H}, \mathrm{H}_{\mathrm{BC}}, \mathrm{E}_{\mathrm{BC}}, \mathrm{k}, \mathrm{T}$ e $\sigma$.

Inicialmente, avaliou-se a relação entre as propriedades da base cimentada $\left(\mathrm{H}_{\mathrm{BC}}\right.$ e $\left.\mathrm{E}_{\mathrm{BC}}\right)$. Estudando a superfície de tensões formada pela variação desses parâmetros (Figura 1), observou-se que a função de $\mathrm{H}_{\mathrm{BC}}$ apresentava comportamento parabólico enquanto a função de $E_{B C}$ tendia a ser linear. Westergaard (1927) apresenta o raio de rigidez relativo $(\ell)$ como uma relação entre a espessura de uma placa, o módulo de elasticidade de seu material e o módulo de reação de seu sistema de apoio. Este termo é recorrente em diversos trabalhos baseados na teoria da elasticidade, como nos coeficientes de Bradbury (1938) ou mesmo no modelo de tensão em placas submetidas a gradientes térmicos de Silfwerbrand (1994). Assim, tem-se o raio de rigidez relativo definido como:

$$
\ell=\sqrt[4]{\frac{E_{B C} \cdot H_{B C}{ }^{3}}{12 \cdot k \cdot\left(1-\mu^{2}\right)}}
$$

em que, $\quad \ell$ : raio de rigidez relativo $(\mathrm{m})$;

$E_{B C}$ : módulo de elasticidade da base cimentada (MPa);

$H_{B C}:$ espessura da base cimentada $(\mathrm{m})$;

$\mu$ : coeficiente de Poisson da base cimentada;

$k$ : módulo de reação do sistema de apoio $(\mathrm{MPa} / \mathrm{m})$.

Análises mais aprofundadas dessa relação, dentro do projeto fatorial, levaram à adoção de uma segunda variável transformada, caracterizada como o quadrado do raio de rigidez relativo $\left(L=\ell^{2}\right)$. Prosseguiu-se, então, com a análise das relações entre as variáveis $(H$,

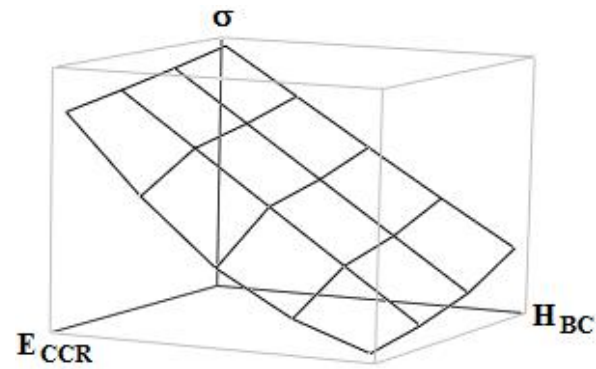

Figura 1. Comportamento da tensão em função das características da base cimentada

L, k, T e $\sigma$ ). Observa-se, da Figura 2, que há uma relação não linear entre $\sigma$ e as variáveis $\mathrm{H}$ e L. A relação entre a tensão e o gradiente térmico se dá por meio de uma função linear, resultado do próprio modelo de elementos finitos. Análises mais aprofundadas entre a tensão e o módulo de reação do subleito (k) (Figura 2) mostraram que a relação entre elas é não linear, apesar da aparência contrária suposta na Figura 3.

Montgomery e Runger (2003) e Hair Jr. et al. (2005) exemplificam que polinômios são boas alternativas para se obter a linearidade entre duas variáveis. A interação entre as variáveis pode ser obtida com a adição de termos cruzados entre essas variáveis no modelo. Assim, optou-se por utilizar polinômios de segundo grau para representar as relações nãolineares. No tocante aos termos cruzados, avaliou-se sobre quais termos dos polinômios eles estariam presentes e concluiu-se que a melhor aproximação seria obtida com o cruzamento de todos os termos.

\subsubsection{Execução das regressões múltiplas para estruturas com base cimentada}

Dessa análise preliminar dos dados obtidos pelas si-
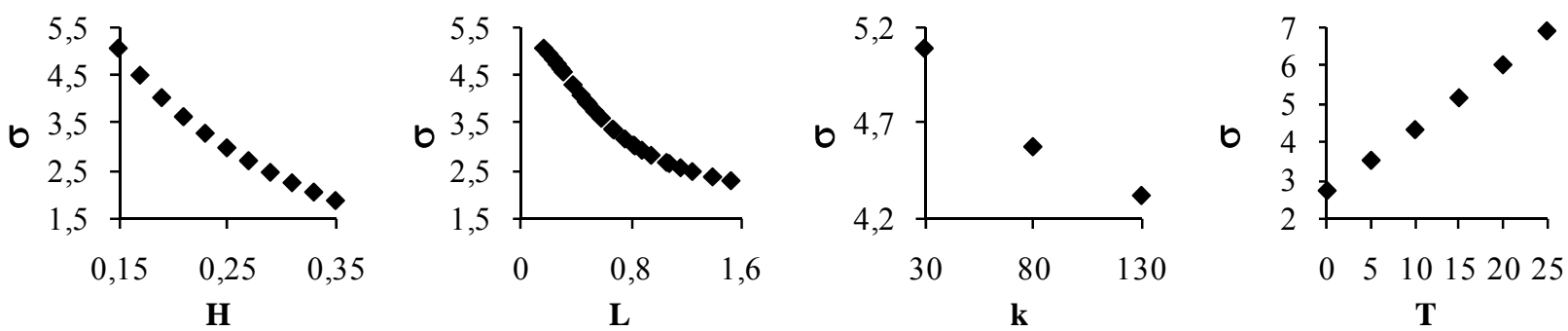

Figura 2. Gráficos de dispersão para análise isolada entre cada regressor e a variável dependente ( $\sigma$ em MPa, $H$ em m, L em m², k em $\mathrm{MPa} / \mathrm{m}$ e $\mathrm{T}^{\mathrm{em}}{ }^{\circ} \mathrm{C}$ )
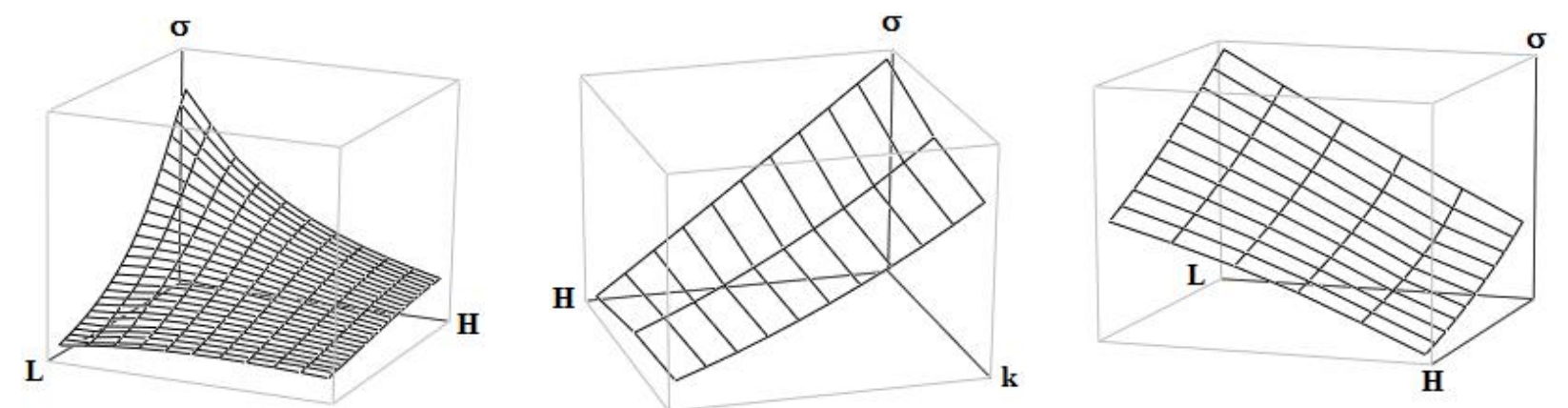

Figura 3. Relação entre os regressores $(H, L, k, T)$ e a variável dependente $(\sigma)$ 
mulações, conclui-se que a tensão seria uma função dos parâmetros estruturais multiplicada por um fator que representaria a relação entre a tensão observada com gradiente térmico e a tensão encontrada na mesma estrutura com gradiente térmico nulo:

$$
\sigma=f_{1}(H, L, k, T=0) \cdot f_{2}(H, L, k, T)
$$

sendo que para uma estrutura analisada $i$, submetida a um gradiente térmico $t$ :

$$
f_{2, i, t}=\frac{\sigma_{i, t}}{\sigma_{i, t=0}}
$$

Optou-se, também, por utilizar um software presente na enorme maioria dos computadores, o MS-Excel, para a execução das regressões lineares múltiplas. Apesar de ser de fácil utilização, o software em questão possui a limitação máxima de dezesseis regressores a serem analisados simultaneamente. A adoção do cruzamento de todos os termos da função $f_{1}$ resultou no tratamento de 26 regressores. Dada a limitação do software de análise escolhido, executou-se a regressão em duas etapas:

- Análise e regressão de $f_{1}$ em função de cada valor individual de $\mathrm{k}(30 \mathrm{MPa} / \mathrm{m}, 80 \mathrm{MPa} / \mathrm{m}$ e 130 $\mathrm{MPa} / \mathrm{m}$ ), mantendo-se as demais variáveis constantes, obtendo-se, assim os diversos coeficientes de regressão $\mathrm{C}_{\mathrm{i}}(\mathrm{k})$

$$
\begin{aligned}
f_{1}(k) & =\mathrm{C}_{1}(k) H^{2} L^{2}+\mathrm{C}_{2}(k) H^{2} L+\mathrm{C}_{3}(k) H^{2}+ \\
& +\mathrm{C}_{4}(k) H L^{2}+\mathrm{C}_{5}(k) H L+\mathrm{C}_{6}(k) H+ \\
& +\mathrm{C}_{7}(k) L^{2}+\mathrm{C}_{8}(k) L+\mathrm{C}_{9}(k)
\end{aligned}
$$

- Posterior regressão de cada $\mathrm{C}_{\mathrm{i}}(\mathrm{k})$ em função dos módulos de reação do subleito, obtendo-se, assim os coeficientes $\mathrm{C}_{\mathrm{i}, 1}, \mathrm{C}_{\mathrm{i}, 2}$ e $\mathrm{C}_{\mathrm{i}, 3}$

$$
\mathrm{C}_{\mathrm{i}}=\mathrm{C}_{\mathrm{i}, 1} \cdot k^{2}+\mathrm{C}_{\mathrm{i}, 2} \cdot k+\mathrm{C}_{\mathrm{i}, 3}
$$

sendo k: módulo de reação do subleito $[\mathrm{MPa} / \mathrm{m}]$

Tem-se, assim, a equação do primeiro termo da função da tensão como:

$$
\begin{aligned}
f_{1} & =\mathrm{C}_{1} H^{2} L^{2}+\mathrm{C}_{2} H^{2} L+\mathrm{C}_{3} H^{2}+\mathrm{C}_{4} H L^{2}+ \\
& +\mathrm{C}_{5} H L+\mathrm{C}_{6} H+\mathrm{C}_{7} L^{2}+\mathrm{C}_{8} L+\mathrm{C}_{9}
\end{aligned}
$$

Analogamente, modelou-se o termo dependente do gradiente térmico, chegando-se à seguinte função:

$$
\begin{aligned}
f_{2} & =\mathrm{C}_{10} H^{2} L^{2} T+\mathrm{C}_{11} H^{2} L T+\mathrm{C}_{12} H^{2} T+\mathrm{C}_{13} H L^{2} T+ \\
& +\mathrm{C}_{14} H L T+\mathrm{C}_{15} H T+\mathrm{C}_{16} L^{2} T+\mathrm{C}_{17} L T+\mathrm{C}_{18} T+1
\end{aligned}
$$

Na Tabela 3 são apresentados os coeficientes de regressão $\mathrm{C}_{1,1}$ a $\mathrm{C}_{18,3}$ utilizados para calcular a tensão em pavimentos com bases cimentadas. A equação final, decorrente do agrupamento dos termos $f_{1}$ e $f_{2}$, retornando a variável $\mathrm{L}$ para $\ell^{2}$ passa a ser a seguinte:

$$
\begin{aligned}
\sigma & =\left[\left(\mathrm{C}_{1} \ell^{4}+\mathrm{C}_{2} \ell^{2}+\mathrm{C}_{3}\right) \cdot H^{2}+\left(\mathrm{C}_{4} \ell^{4}+\mathrm{C}_{5} \ell^{2}+\mathrm{C}_{6}\right) \cdot\right. \\
& \left.\cdot H+\left(\mathrm{C}_{7} \ell^{4}+\mathrm{C}_{8} \ell^{2}+\mathrm{C}_{9}\right)\right] \cdot\left[\left(\mathrm{C}_{10} \ell^{4}+\mathrm{C}_{11} \ell^{2}+\mathrm{C}_{12}\right) \cdot\right. \\
& \cdot H^{2} T+\left(\mathrm{C}_{13} \ell^{4}+\mathrm{C}_{14} \ell^{2}+\mathrm{C}_{15}\right) \cdot H T+\left(\mathrm{C}_{16} \ell^{4}+\mathrm{C}_{17} \ell^{2}+\right. \\
& \left.\left.+\mathrm{C}_{18}\right) \cdot T+1\right]
\end{aligned}
$$

sendo, $\quad C_{i}$ : calculado como apresentado na equação 5;

$\ell$ : raio de rigidez relativo da base cimentada $[\mathrm{m}]$;

$H$ : espessura da placa de CCP [m];

$T$ : gradiente térmico entre o topo e o fundo da placa $\left[{ }^{\circ} \mathrm{C}\right]$.

\subsection{Pavimentos com bases granulares}

O projeto fatorial de pavimentos em concreto de cimento Portland com bases granulares resultou na análise de 330 casos. Seguindo o mesmo processo apresentado para a análise dos casos com bases cimenta-

\begin{tabular}{|c|c|c|c|c|c|c|c|}
\hline $\mathrm{Ci}$ & $C_{i, 1}$ & $C_{i, 2}$ & $C_{i, 3}$ & $\mathrm{Ci}$ & $C_{i, 1}$ & $C_{i, 2}$ & $C_{i, 3}$ \\
\hline $\mathrm{C}_{1}$ & $-0,00252$ & 2,217 & 13,585 & $\mathrm{C}_{10}$ & 0,000044 & 0,0335 & $-0,162$ \\
\hline $\mathrm{C}_{2}$ & 0,00542 & $-1,921$ & $-121,44$ & $\mathrm{C}_{11}$ & $-0,0000683$ & 0,0285 & 0,609 \\
\hline $\mathrm{C}_{3}$ & 0,001114 & $-0,356$ & 89,90 & $\mathrm{C}_{12}$ & 0,0000377 & $-0,00608$ & $-1,287$ \\
\hline $\mathrm{C}_{4}$ & 0,001513 & $-1,321$ & $-7,929$ & $\mathrm{C}_{13}$ & $-0,0000307$ & $-0,0281$ & 0,1873 \\
\hline $\mathrm{C}_{5}$ & $-0,003388$ & 1,2023 & 75,21 & $\mathrm{C}_{14}$ & 0,0000326 & $-0,01567$ & $-0,4056$ \\
\hline $\mathrm{C}_{6}$ & $-0,000789$ & 0,247 & $-61,86$ & $\mathrm{C}_{15}$ & $-0,0000345$ & 0,008453 & 0,6752 \\
\hline $\mathrm{C}_{7}$ & $-0,00022$ & 0,1916 & 1,123 & $\mathrm{C}_{16}$ & 0,0000068 & 0,00675 & $-0,0465$ \\
\hline $\mathrm{C}_{8}$ & 0,000528 & $-0,1886$ & $-11,73$ & $\mathrm{C}_{17}$ & $-0,0000026$ & 0,002216 & 0,06294 \\
\hline $\mathrm{C}_{9}$ & 0,000162 & $-0,04921$ & 12,01 & $\mathrm{C}_{18}$ & 0,0000028 & $-0,000788$ & $-0,0451$ \\
\hline
\end{tabular}
das, avaliou-se a relação entre os regressores $(\mathrm{H}, \mathrm{k}$ e T) e a variável dependente $(\sigma)$.

Da análise das dispersões (Figura 4), conclui-se que as variações da tensão em função da espessura da placa $(\mathrm{H})$ e do módulo de reação do sistema de apoio (k) não são lineares, enquanto a variação de $\sigma$ em função do gradiente térmico é linear. Observa-se que a variação da tensão em decorrência de $\mathrm{k}$ é bastante pequena (a escala vertical para a tensão no caso não parte de zero). De modo a analisar a relação cruzada entre os regressores, construíram-se gráficos de superfícies, apresentados na Figura 5.

Tabela 3. Coeficientes de regressão para estruturas com base cimentada 

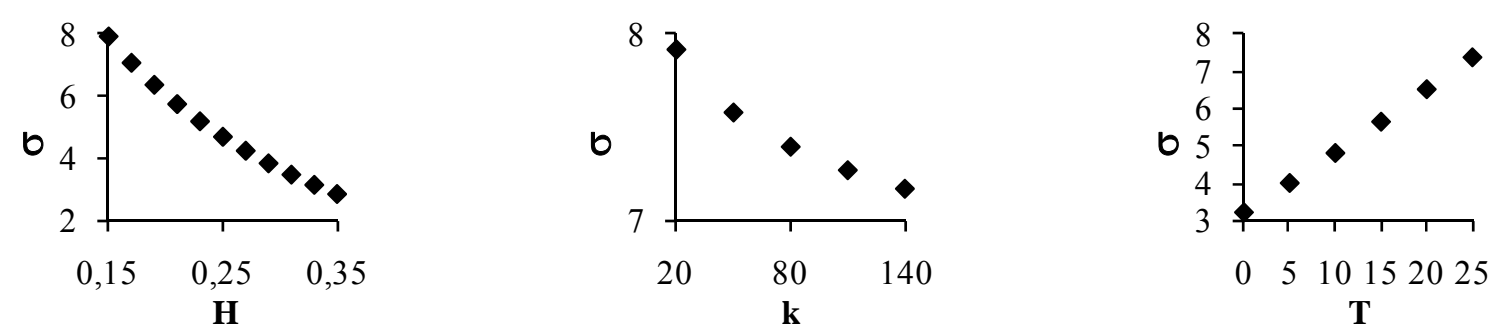

Figura 4. Gráficos de dispersões dos regressores dos pavimentos com bases granulares $\left(\sigma\right.$ em MPa, $\mathrm{Hem} \mathrm{m}, \mathrm{k} \mathrm{em} \mathrm{MPa/m} \mathrm{e} \mathrm{T} \mathrm{em}{ }^{\circ} \mathrm{C}$ )
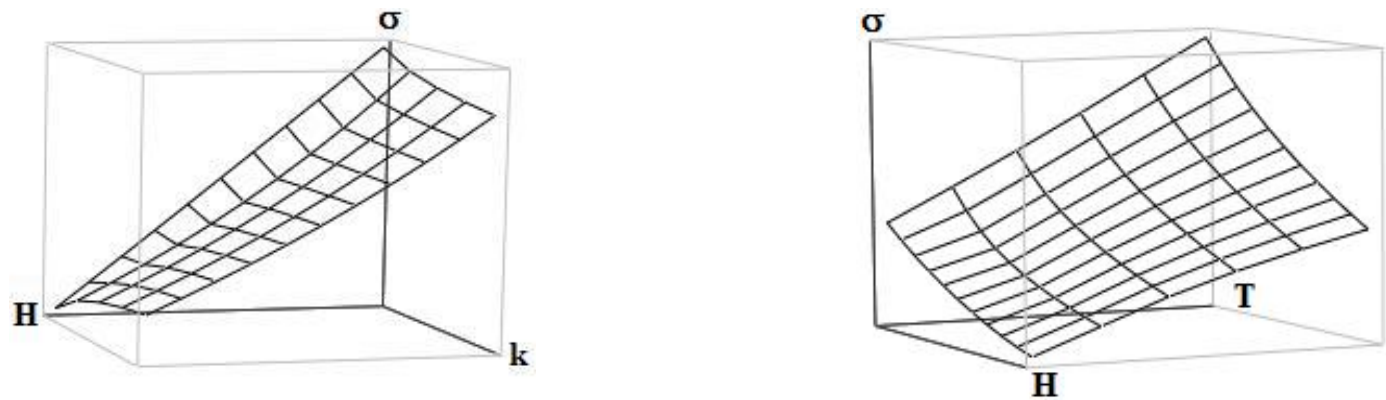

Figura 5. Relacionamento entre os parâmetros

\subsubsection{Execução das regressões múltiplas para estruturas com base granular}

Decidiu-se por segregar a função para cômputo das tensões em duas parcelas, do mesmo modo como apresentado nos casos com base cimentada (equação 2). Para o tratamento da variação da tensão em função de $\mathrm{H}$ e $\mathrm{k}$, tendo o gradiente térmico nulo, utilizou-se a seguinte função:

$$
f_{1}=10^{C_{1}} \cdot H^{C_{2}} \cdot k^{C_{3}}
$$

Por conveniência das regressões, transformou-se a função acima em uma função linear através da função logarítmica:

$$
\begin{aligned}
\log _{10}\left(f_{1}\right) & =\log _{10}\left(10^{C_{1}} \cdot H^{C_{2}} \cdot k^{C_{3}}\right)= \\
& =C_{1}+C_{2} \cdot \log _{10}(H)+C_{3} \cdot \log _{10}(k)
\end{aligned}
$$

A regressão da segunda parcela da equação foi efetuada objetivando ter uma função similar à função do fator dependente do gradiente térmico definida para os casos com base cimentada:

$$
\begin{aligned}
f_{2} & =\mathrm{C}_{4} H^{2} k^{2} T+\mathrm{C}_{5} H^{2} k T+\mathrm{C}_{6} H^{2} T+\mathrm{C}_{7} H k^{2} T+ \\
& +\mathrm{C}_{8} H k T+\mathrm{C}_{9} H T+\mathrm{C}_{10} k^{2} T+\mathrm{C}_{11} k T+\mathrm{C}_{12} T+1
\end{aligned}
$$

Com a execução das regressões, foram obtidos coeficientes apresentados na Tabela 4 a seguir:

$$
\begin{aligned}
\sigma & =10^{C_{1}} \cdot H^{C_{2}} \cdot k^{C_{3}} \cdot\left[\mathrm{C}_{4} H^{2} k^{2} T+\mathrm{C}_{5} H^{2} k T+\right. \\
& +\mathrm{C}_{6} H^{2} T+\mathrm{C}_{7} H k^{2} T+\mathrm{C}_{8} H k T+\mathrm{C}_{9} H T+ \\
& \left.+\mathrm{C}_{10} k^{2} T+\mathrm{C}_{11} k T+\mathrm{C}_{12} T+1\right]
\end{aligned}
$$

\subsection{Análise da aderência estatística dos modelos propostos}

A aderência das equações propostas foi testada contra a resposta original das tensões obtidas por meio do
Tabela 4. Coeficientes de regressão para estruturas com base granular

\begin{tabular}{ccccc}
\hline Coeficiente & Valor & & Coeficiente & Valor \\
\cline { 1 - 2 } \cline { 5 - 5 }$C_{1}$ & $-0,384$ & & $C 7$ & $-3,62 \mathrm{E}-05$ \\
$C_{2}$ & $-1,482$ & & $C 8$ & $8,94 \mathrm{E}-03$ \\
$C_{3}$ & $-0,174$ & & $C 9$ & $4,72 \mathrm{E}-01$ \\
$\mathrm{C}_{4}$ & $3,90 \mathrm{E}-05$ & & $\mathrm{C} 10$ & $3,58 \mathrm{E}-06$ \\
$\mathrm{C}_{5}$ & $-7,00 \mathrm{E}-03$ & & $\mathrm{C} 11$ & $-8,75 \mathrm{E}-04$ \\
$\mathrm{C}_{6}$ & $-9,60 \mathrm{E}-01$ & & $\mathrm{C} 12$ & $-1,66 \mathrm{E}-02$ \\
\hline
\end{tabular}

ILSL2. Hair Jr. et al (2005) apresentam que, para uma amostra Y com $n$ dados observados $\left(y_{i}\right)$, com média $(\bar{y})$ e modelados por uma regressão $\hat{y}_{i} \operatorname{com}(m-1)$ graus de liberdade $(\mathrm{gl})$, tem-se que os erros quadrados são definidos por:

$$
\begin{gathered}
\mathrm{SQ}_{\text {Residuos }}=\sum\left(y_{i}-\hat{y}_{i}\right)^{2} \\
\mathrm{SQ}_{\text {Regressão }}=\sum\left(\bar{y}-y_{i}\right)^{2} \\
\mathrm{SQ}_{\text {Total }}=\mathrm{SQ}_{\text {Residuos }}+\mathrm{SQ}_{\text {Regressão }}
\end{gathered}
$$

Os primeiros dois parâmetros a expressarem a aderência da regressão proposta aos valores iniciais são o coeficiente de determinação $\left(\mathrm{R}^{2}\right)$ e o Erro Padrão, além do F de significação:

$$
\begin{gathered}
\mathrm{R}^{2}=\frac{\mathrm{SQ}_{\text {Regressão }}}{\mathrm{SQ}_{\text {Total }}} \\
\mathrm{R}_{\text {Ajustado }}^{2}=1-\left[\left(\frac{n-1}{n-m-1}\right) \cdot\left(1-\mathrm{R}^{2}\right)\right]
\end{gathered}
$$


Tabela 5. Parâmetros estatísticos dos modelos de regressão

\begin{tabular}{|c|c|c|c|c|c|c|c|c|c|}
\hline \multicolumn{5}{|c|}{ Equação 8} & \multicolumn{5}{|c|}{ Equação 12} \\
\hline $\mathrm{n}$ & \multicolumn{3}{|c|}{4950} & & $\mathrm{n}$ & \multicolumn{2}{|c|}{330} & & \\
\hline$\sigma_{\text {média }}$ & \multicolumn{3}{|c|}{3,0208} & & $\sigma_{\text {média }}$ & \multicolumn{2}{|c|}{3,4878} & & \\
\hline $\mathrm{R}^{2}$ & \multicolumn{3}{|c|}{0,9978} & & $\mathrm{R}^{2}$ & \multicolumn{2}{|c|}{0,9981} & & \\
\hline $\mathrm{R}_{\text {ajustado }}^{2}$ & \multicolumn{3}{|c|}{0,9978} & & $\mathrm{R}_{\text {ajustado }}^{2}$ & \multicolumn{2}{|r|}{0,9980} & & \\
\hline Erro Padrão & \multicolumn{3}{|c|}{0,06585} & & Erro Padrão & \multirow{2}{*}{\multicolumn{2}{|c|}{0,0678}} & & \\
\hline ANOVA & & & & & ANOVA & & & & \\
\hline & SQ & $\mathrm{gl}$ & MS & $\mathrm{F}$ & & SQ & gl & MS & $\mathrm{F}$ \\
\hline Regressão & 9575 & 53 & 180,660 & 41236 & Regressão & 786,75 & 11 & 71,5227 & 15085 \\
\hline Resíduo & 21,45 & 4896 & 0,00438 & & Resíduo & 1,5077 & 318 & 0,00474 & \\
\hline Total & 9597 & 4949 & & & Total & 788,23 & 329 & & \\
\hline
\end{tabular}

$$
\begin{gathered}
\text { Erro Padrão }=\sqrt{\frac{\mathrm{SQ}_{\text {Resíduos }}}{n-2}} \\
\qquad \begin{array}{c}
\mathrm{F}=\frac{\frac{\mathrm{SQ}_{\text {Regressão }}}{m-1}}{\frac{\mathrm{SQ}_{\text {Residuos }}}{n-m-2}}
\end{array}
\end{gathered}
$$

A aplicação das equações acima nos dados originais calculados pelo MEF e avaliados pelas equações 8 e 12 resultou nos parâmetros apresentados na Tabela 5 . Os valores dos parâmetros estatísticos de ambas as funções garantem sua aderência às simulações do MEF que as gerou.

\subsection{Confronto entre os modelos propostos e métodos recentemente publicados}

Avaliaram-se as diferenças e similaridades entre as equações propostas e dois métodos de dimensionamento recentemente publicados: a Instrução de Projeto (IP-07), da Secretaria de Infraestrutura Urbana da Prefeitura de São Paulo (PMSP, 2004) e o Mechanistic-Empirical Pavement Design Guide (MEPDG) da AASHTO (NCHRP, 2003). O IP-07, assim como este trabalho, foi desenvolvido a partir de simulações numéricas executadas no ILSL2. Seu coeficiente de determinação é de 0,9905 o que demonstra uma grande correlação ao MEF original. Sua estrutura de equações, que não consideram termos cruzados entre seus

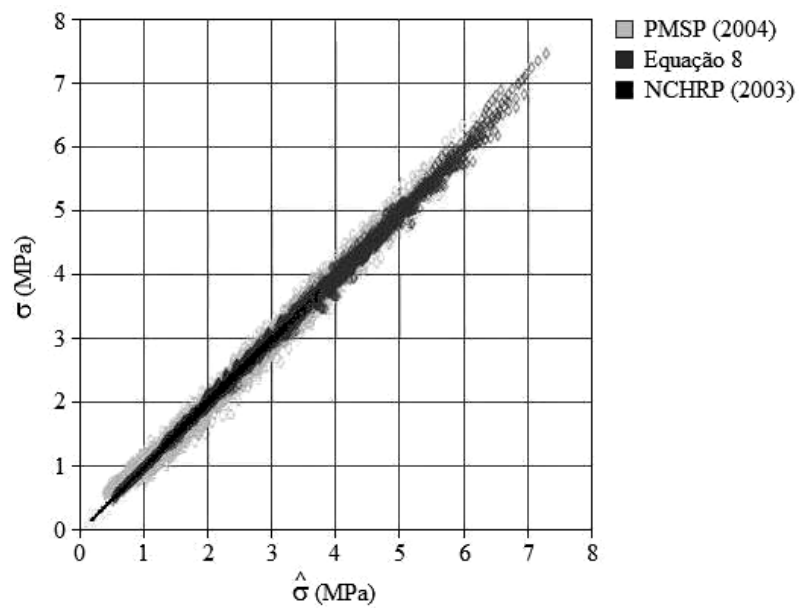

Figura 6. Confronto entre os diversos modelos parâmetros (Rodolfo e Balbo, 2002; PMSP, 2004), colabora para a maior dispersão de pontos apresentada na Figura 6.

O MEPDG foi desenvolvido a partir do ISLAB2000, que possui a mesma estrutura analítica do programa de elementos finitos ILSL2. É um método de dimensionamento fechado, ou seja, suas equações (baseadas em redes neurais) não são acessíveis. Ainda assim, pode ser obtido de sua documentação que uma amostra de teste da rede neural apresentou coeficiente de determinação de 0,9982 e valores máximos de tensão de aproximadamente $3,8 \mathrm{MPa}$ (NCHRP, 2003).

Os resultados obtidos pela Equação 8 apresentaram dispersões muito inferiores àquelas observadas no IP07 em decorrência da adoção de termos cruzados entre os parâmetros. A comparação entre os resultados calculados da Equação 8 com o MEPDG mostra que, para tensões situadas até $2 \mathrm{MPa}$, seus valores são praticamente idênticos. Um pequeno aumento na dispersão da Equação 8 é observado no intervalo de $2 \mathrm{MPa}$ a 3 $\mathrm{MPa}$ de tensão, porém esse aumento não é suficiente grande a ponto de alterar a conclusão de que os modelos conduzem a resultados idênticos.

\section{APLICAÇÃO DOS MODELOS DESENVOLVIDOS}

Nesta etapa, apresenta-se a aplicação dos modelos em um caso de rodovia recentemente construída no Estado de São Paulo: a pista descendente da Rodovia dos Imigrantes. Para o cálculo do número de repetição de carga admitido, utilizou-se o modelo apresentado por Cervo et al. (2005) e o consumo total à fadiga foi computado de acordo com a regra de Miner (1945). Desse modo, para um caso $i$ :

$$
\begin{gathered}
\mathrm{N}_{\mathrm{adm}, i}=\left(10^{\left.25,858-25,142 \cdot \frac{\sigma_{i}}{\mathrm{f}_{\mathrm{ct}, \mathrm{f}}}\right) \cdot\left(\frac{\sigma_{i}}{\mathrm{f}_{\mathrm{ct}, \mathrm{f}}}\right)^{4,20231}}\right. \\
\mathrm{CRF}=\sum \frac{\mathrm{N}_{\mathrm{proj}, i}}{\mathrm{~N}_{\mathrm{adm}, i}} \leq 100 \%
\end{gathered}
$$

sendo, 
$N_{a d m, i}$ : número de repetições de carga admissível;

$N_{\text {proj: }}$ número de repetições de carga estimado em projeto;

$\sigma_{i}$ : tensão calculada [Mpa];

$f_{c t, f}$ : resistência à tração na flexão de projeto do CCP.

Melo et al. (2007) apresentaram trabalho acerca da construção do pavimento rígido da pista descendente da Rodovia dos Imigrantes. Segundo os autores, o tráfego solicitante para um período de projeto de 20 anos seria de $3,24 \times 10^{7}$ repetições de um eixo simples de rodas duplas de $80 \mathrm{kN}$. O pavimento conta com base de concreto compactado com rolo de $0,10 \mathrm{~m}$ de espessura e sub-base granular de $0,15 \mathrm{~m}$ de espessura. $\mathrm{O}$ subleito apresentaria CBR igual ou superior a $10 \%$. O coeficiente de recalque do sistema sub-base + subleito adotado foi de $64 \mathrm{Mpa} / \mathrm{m}$. A resistência média do concreto à tração na flexão considerada foi de 4,5 Mpa. A adoção do método da AASHTO/1993 resultou em uma placa de concreto com 0,22 $\mathrm{m}$ de espessura, verificada posteriormente pelo método da PCA/1966.
Propôs-se, nesse momento, uma verificação desse projeto, de acordo com as informações apresentadas por Melo et al. (2007) e os conceitos discutidos neste trabalho. Para a caracterização do gradiente térmico na placa de concreto, adotaram-se os valores sugeridos pela PMSP (2004) para cada uma das estações do ano. O tráfego foi uniformemente distribuído ao longo do ano e ao longo do dia. Essa análise resultou em uma espessura de placa de 0,24 m (9\% superior ao projetado inicialmente para a rodovia) e consumo da resistência à fadiga total de $54 \%$. Os resultados da análise são apresentados na Tabela 6 . Nos túneis, onde o gradiente térmico é nulo, o projeto resultaria em espessura de $0,15 \mathrm{~m}$, contra os 0,22 $\mathrm{m}$ de espessura adotada no projeto. Pode-se observar que o $42 \%$ do consumo da resistência à fadiga se dá por apenas 2,08\% do tráfego, caracterizado por aquele que ocorre na primavera, das 14:00 às 16:00 horas. Tem-se nesse ponto caracterizada a grande dependência do projeto de um pavimento em concreto com as cargas ambientais. Em se adotando base granular, as mesmas espes-

Tabela 6. Cálculo das tensões e dos consumos da resistência à fadiga individuais

\begin{tabular}{|c|c|c|c|c|c|c|c|}
\hline & Faixa Horária & $\begin{array}{c}\text { \% Tráfego } \\
\text { Previsto }\end{array}$ & $\begin{array}{c}T \\
\left({ }^{\circ} \mathrm{C}\right)\end{array}$ & $\begin{array}{c}\sigma \\
(M P a)\end{array}$ & $\sigma / f t$ & Nadm & CRF \\
\hline Gradiente Nulo & variável & $60,42 \%$ & 0,0 & 1,581 & 0,351 & $1,31 \mathrm{E}+15$ & $0 \%$ \\
\hline \multirow{10}{*}{ Primavera } & 9 & $1,04 \%$ & 1,3 & 1,766 & 0,392 & $1,93 \mathrm{E}+14$ & $0 \%$ \\
\hline & 10 & $1,04 \%$ & 3,8 & 2,135 & 0,474 & $3,70 \mathrm{E}+12$ & $0 \%$ \\
\hline & 11 & $1,04 \%$ & 6,3 & 2,505 & 0,557 & $6,23 \mathrm{E}+10$ & $0 \%$ \\
\hline & 12 & $1,04 \%$ & 8,8 & 2,874 & 0,639 & $9,57 \mathrm{E}+08$ & $0 \%$ \\
\hline & 13 & $1,04 \%$ & 11,3 & 3,244 & 0,721 & $1,37 \mathrm{E}+07$ & $2 \%$ \\
\hline & 14 & $1,04 \%$ & 12,5 & 3,429 & 0,762 & $1,60 \mathrm{E}+06$ & $21 \%$ \\
\hline & 15 & $1,04 \%$ & 12,5 & 3,429 & 0,762 & $1,60 \mathrm{E}+06$ & $21 \%$ \\
\hline & 16 & $1,04 \%$ & 10,4 & 3,121 & 0,693 & $5,68 \mathrm{E}+07$ & $1 \%$ \\
\hline & 17 & $1,04 \%$ & 6,3 & 2,505 & 0,557 & $6,23 \mathrm{E}+10$ & $0 \%$ \\
\hline & 18 & $1,04 \%$ & 2,1 & 1,889 & 0,420 & $5,26 \mathrm{E}+13$ & $0 \%$ \\
\hline \multirow{11}{*}{ Verão } & 9 & $1,04 \%$ & 1,2 & 1,751 & 0,389 & $2,26 \mathrm{E}+14$ & $0 \%$ \\
\hline & 10 & $1,04 \%$ & 3,5 & 2,091 & 0,465 & $5,99 \mathrm{E}+12$ & $0 \%$ \\
\hline & 11 & $1,04 \%$ & 5,8 & 2,431 & 0,540 & $1,42 \mathrm{E}+11$ & $0 \%$ \\
\hline & 12 & $1,04 \%$ & 8,1 & 2,771 & 0,616 & $3,11 \mathrm{E}+09$ & $0 \%$ \\
\hline & 13 & $1,04 \%$ & 10,4 & 3,111 & 0,691 & $6,36 \mathrm{E}+07$ & $1 \%$ \\
\hline & 14 & $1,04 \%$ & 11,5 & 3,281 & 0,729 & $8,93 \mathrm{E}+06$ & $4 \%$ \\
\hline & 15 & $1,04 \%$ & 11,5 & 3,281 & 0,729 & $8,93 \mathrm{E}+06$ & $4 \%$ \\
\hline & 16 & $1,04 \%$ & 10,1 & 3,068 & 0,682 & $1,04 \mathrm{E}+08$ & $0 \%$ \\
\hline & 17 & $1,04 \%$ & 7,2 & 2,643 & 0,587 & $1,31 \mathrm{E}+10$ & $0 \%$ \\
\hline & 18 & $1,04 \%$ & 4,3 & 2,218 & 0,493 & $1,49 E+12$ & $0 \%$ \\
\hline & 19 & $1,04 \%$ & 1,4 & 1,793 & 0,398 & $1,44 \mathrm{E}+14$ & $0 \%$ \\
\hline \multirow{9}{*}{ Outono } & 10 & $1,04 \%$ & 1,0 & 1,729 & 0,384 & $2,85 \mathrm{E}+14$ & $0 \%$ \\
\hline & 11 & $1,04 \%$ & 3,0 & 2,024 & 0,450 & $1,23 \mathrm{E}+13$ & $0 \%$ \\
\hline & 12 & $1,04 \%$ & 5,0 & 2,320 & 0,516 & $4,87 \mathrm{E}+11$ & $0 \%$ \\
\hline & 13 & $1,04 \%$ & 7,0 & 2,616 & 0,581 & $1,80 \mathrm{E}+10$ & $0 \%$ \\
\hline & 14 & $1,04 \%$ & 8,0 & 2,763 & 0,614 & $3,38 \mathrm{E}+09$ & $0 \%$ \\
\hline & 15 & $1,04 \%$ & 8,0 & 2,763 & 0,614 & $3,38 \mathrm{E}+09$ & $0 \%$ \\
\hline & 16 & $1,04 \%$ & 6,7 & 2,566 & 0,570 & $3,12 \mathrm{E}+10$ & $0 \%$ \\
\hline & 17 & $1,04 \%$ & 4,0 & 2,172 & 0,483 & $2,47 \mathrm{E}+12$ & $0 \%$ \\
\hline & 18 & $1,04 \%$ & 1,3 & 1,778 & 0,395 & $1,70 \mathrm{E}+14$ & $0 \%$ \\
\hline
\end{tabular}


suras seriam resultadas.

Deve-se atentar, ainda, que a espessura calculada é motivada não apenas pelo resultado dos gradientes térmicos, mas também pela adoção de um novo modelo de fadiga do CCP. Caso fosse adotado o modelo de fadiga proposto pela PCA/84 (que é idêntico ao modelo de 1966 , com alteração no tramo no qual a relação entre as tensões $-\sigma$ e fct,f - possui valores de 0,45 a $0,55)$, o mesmo projeto levaria em uma placa com espessura de 0,30 m e CCP com 5,5 Mpa de resistência à tração na flexão. Ressalta-se que concretos com resistência dessa magnitude apresentam elevado consumo de ligante hidráulico com reflexos na ocorrência de retração plástica e hidráulica.

\section{CONCLUSÕES}

Este trabalho apresentou os fundamentos para a determinação de novos modelos para o cálculo de tensões em placas de concreto de pavimentos submetidas a carregamentos rodoviários e ambientais, justificando-se a adoção de cada passo sob o ponto de vista da análise multivariada de dados. As equações apresentadas, tanto para pavimentos com bases cimentadas quanto para pavimentos com bases granulares, tiveram seus parâmetros estatísticos calculados e foram confrontadas com dois outros métodos modernos de dimensionamento de pavimentos em concreto.

Desse confronto, conclui-se que as equações apresentadas são estatisticamente melhores que aquelas adotadas pelo IP-07 da PMSP em função da incorporação de novos termos cruzados nas mesmas. Em se comparando seus resultados com o modelo de redes neurais presente no MEPDG, conclui-se que ambos são estatisticamente idênticos em um mesmo intervalo de tensões. A aplicação das novas equações em conjunto com um novo modelo de fadiga do CCP em um projeto real resultou em espessuras superiores nos trechos sujeitos a gradientes térmicos e em espessuras muito inferiores nos trechos de túneis do que as espessuras originalmente adotadas no projeto real. A variação de apenas $2 \mathrm{~cm}$ na espessura da placa dimensionada pelo novo método e a espessura original é devido principalmente à adoção de um novo modelo de fadiga, posto que a espessura calculada em função dos gradientes térmicos e o modelo de fadiga da PCA/84 resultou em espessuras muito maiores e a necessidade de alterações nas características do CCP.

Constata-se, portanto, a necessidade do desenvolvimento de um método de dimensionamento que leve em consideração as características ambientais de cada uma das regiões do Brasil e a distribuição das cargas rodoviárias ao longo dos diferentes períodos do dia. A normatização dos projetos de pavimentos rodoviários em concreto de cimento Portland permitirá melhores decisões nos investimentos de infraestrutura bem como melhorias em sua durabilidade quanto às ações estruturais analisadas. O Brasil possui conhecimento e capacidade técnica suficiente para dar um salto de qualidade em seus projetos de pavimentos de concreto de cimento Portland.

\section{REFERÊNCIAS BIBLIOGRÁFICAS}

Armaghani, J. M.; T. J. Larsen e L. Smith (1987) Temperature response of concrete pavement. Transportation Research Record. Transportation Research Board, Washington, D.C., 1121, p. 23-33.

Balbo, J. T. e A. A. Severi (2002) Thermal gradients in concrete pavements in tropical (hot-wet) environment: an experimental appraisal. Transportation Research Record. Transportation Research Board, Washington, D.C., 1809, p. 12-22.

Bradradbury, R. D. (1938) Reinforced Concrete Pavement. Wire Reinforced Institute, Washington, D.C.

Cervo, T. C.; A. A. Severi; M. P. Rodolfo; D. S. Pereira e J. T. Balbo (2005) Innovative Aspects of the Concrete Pavement Design Standard for Sao Paulo Highways. In: International Conference on Concrete Pavements, 8, Colorado Springs, 2005. Proceedings. International Society for Concrete Pavements, Colorado Springs. CD-ROM.

Hair Jr., J. F.; R. L. Tatham; R. E. Anderson e W. Black (2005) Análise Multivariada de Dados ( $5^{\mathrm{a}}$ ed.). Bookman, Porto Alegre.

Hall, K. T. (2000) State of the art and practice in rigid pavement design. Transportation in the New Millennium: State of the Art and Future Directions, Perspectives from Transportation Research Board Standing Committees. Transportation Research Board, Washington, D.C., 7 p.. Disponível em $<$ http://onlinepubs.trb.org/Onlinepubs/millennium/00105.pdf $>$. Acesso em: 04 fevereiro 2008.

Huang, Y. H. (2003) Pavement Analysis and Design. (2 ${ }^{\text {nd }}$ Edition) Prentice-Hall, Inc., Englewood Cliffs, USA.

Huang. Y. H. e S. T. Wang (1973) Finite-Element Analysis of Concrete Slabs and its Implications for Rigid Pavement Design. Highway Research Record. Highway Research Board, Washington, D.C., 466, p. 55-69.

Khazanovich, L. e A. M. Ioannnides (1993) Finite Element Analysis of Slabs-On-Grade Using Improved Subgrade Soil Models. In: ASCE Specialty Conference Airport Pavement Innovations Theory to Practice, 1993, Vicksburg. Proceedings. Waterways Experiment Station, Vicksburg, p. 16-30.

Mardia, K. V.; J. T. Kent e J. Bibby (1979). Multivariate Analysis - (Probality and Mathematical Statistics). Academic Press, London.

Melo, D. G. V.; J. J. Zanetti e C. J. Andreucci (2007). Execução do pavimento rígido da Rodovia dos Imigrantes - pista descendente. Concreto \& Construções. IBRACON, São Paulo, n. 45, p. 37-47.

Miner, M. A. (1945) Cumulative Damage in Fatigue. Transactions of the $A S M E$, American Society of Mechanical Engineers, New York, Vol. 67, pp. A159-A164.

Montgomery, D. C. e G. C. Runger (2003) Estatística Aplicada e Probabilidade para Engenheiros ( $2^{\mathrm{a}}$ ed.). LTC, Rio de Janeiro.

National Cooperative Highway Research Program (2003). Guide for Mechanistic-Empirical Design of New and Rehabilated Pavement Structures. Final Report for Project 1-37A. Transportation Research Board, Washington, D.C.

Portland Cement Association (1984). Thickness Design of for Concrete Highways and Streets Pavements. EB209.01P. PCA, Ottawa.

Prefeitura do Município de São Paulo (2004). Secretaria de Infra-Estrutura Urbana. Instruções de Projeto. IP 07 - Dimensionamento de Pavimentos de Concreto. Revisão e atualização das normas de pavimentação. PMSP, São Paulo.

Rodolfo, M. P. e J. T. Balbo (2002) Modelagem de tensões em pavimentos de concreto submetidos a gradientes térmicos e cargas rodoviárias. In: Confederação Nacional dos Transportes; Associação Nacional de Ensino e Pesquisa em Transportes. (Org.). Transporte em Transformação. MAKRON Books, São Paulo, v. 5, p. 101116.

Rodolfo, M. P. e J. T. Balbo (2009) Novo critério de dimensionamento de pavimentos de concreto simples para as cargas ambientais e do tráfego. Revista Pavimentação. ANPET, Rio de Janiero, v. Ano IV, p. 23-33. 
Silfwerbrand, J. (1994) Thermal stress in concrete pavements. In: International Workshop on the Design and Evaluation of Concrete Pavements, 3. Proceedings. CROW, Krumbach, pp. 131 - 40.

Westergaard, H. M. (1927) Analysis of Stresses in Concrete Pavements due to Variations of Temperature. Proceedings. Highway Research Board, Washington, D.C., v. 6, p. 201-215. 